\title{
Methylene Blue in Management of Acute Ifosfamide-Induced Encephalopathy- Case Report and Literature Review
}

\author{
Leszek Kraj ${ }^{1,2 *}$, Joanna Krawczyk ${ }^{1}$, Mateusz Ziarkiewicz ${ }^{1}$, Piotr Boguradzki $^{1}$ and Wiesław Wiktor \\ Jędrzejczak ${ }^{1}$
}

${ }^{1}$ Oncology and Internal Diseases; Medical University of Warsaw, Poland

${ }^{2}$ Medical University of Warsaw, Poland

*Corresponding author: Leszek Kraj, Department of Hematology, Oncology and Internal Diseases Medical University of Warsaw, 02-097 Warszawa, ul. Banacha 1A, Poland, Tel: +48225992898, Fax: +48225991418; E-mail: leszekkraj@gmail.com Received Date: April 09, 2018; Accepted Date: April 28, 2018; Published Date: May 05, 2018

\begin{abstract}
Administration of several chemotherapeutic agents is associated with a significant risk of neurotoxicity. Neurologic adverse reactions are frequently observed after ifosfamide, which metabolites may cause acute ifosfamide-induced encephalopathy (IIE). There is no standard management in this clinical setting.

Herein we report a case of $\mathbf{2 8}$ year old male with a B-cell lymphoma unclassifiable, with features intermediate between diffuse large B-cell lymphoma and classical Hodgkin lymphoma. The patient developed a severe IIE with catatonia, as well as fecal and urinary incontinence during combined systemic ifosfamide-based chemotherapy. The infusion was interrupted; hydration and forced diuresis were implemented. Drugs with known sedative effect on the central nervous system were eliminated. Nevertheless, no improvement was observed. At this point a decision was made to administer methylene blue (MB). Two doses were infused at intervals of 6 hours with fast improvement and elimination of IIE symptoms on the second day after MB infusion. Noteworthy, no toxicity attributable to MB was observed. Described treatment was based on previous case reports and small retrospective series and obtained results fully confirmed the conclusions of these publications.
\end{abstract}

Keywords: Encephalopathy, Ifosfamide; Methylene blue

\section{Introduction}

Ifosfamide is an alkylating agent used to treat different types of malignancies including ovarian, testicular, cervical cancers, lymphomas and sarcomas. Common adverse effects of Ifosfamide include: bone marrow suppression, nausea, and vomiting, hemorrhagic cystitis. However, a specific side effect of Ifosfamide is central nervous system toxicity: Ifosfamide-induced encephalopathy (IIE). The clinical presentation of IIE ranges from mild somnolence, agitation, mental confusion, hallucinations, and deep coma. Although methylene blue (MB) can be used to treat IIE, its mechanism of action remains poorly

Citation: Leszek Kraj, Methylene Blue in Management of Acute Ifosfamide-Induced Encephalopathy-Case Report and Literature Review. J Clin Cases Rep 2018: 1(2): 45-50. DOI: https://doi.org/10.46619/joccr.2018.1-1011 
www.tridhascholars.org | July-2018

understood. We describe patient with B-cell lymphoma unclassifiable who experienced severe IIE and was successful treated with $\mathrm{MB}$, as well as a review of literature about pathophysiology of IIE and the use of MB in its management.

\section{Case Representation}

A 26-year old male first presented with bulky lymphadenopathy, bone pain and tumor of the sternum. The histopathological examination of a supraclavicular node revealed the diagnosis of B-cell lymphoma unclassifiable, with features intermediate between diffuse large B-cell lymphoma and classical Hodgkin lymphoma. The initial stage of the disease was characterized as IVBX according to Cotswolds modification of Ann Arbor staging system, with bulky mediastinal disease (>11 cm in long axis) and disseminated extranodal involvement of the skeleton. The risk according to the International Prognostic Index was low/intermediate.

The first line treatment was CHOP (cyclophosphamide, doxorubicin, vincristin, prednisone) with mediastinal radiotherapy and prophylactic intrathecal chemotherapy. Rituximab was not included in the regimen due to low expression of $\mathrm{CD} 20$ on the surface of tumor cells. The patient achieved partial response, but progressed two months later. The second line treatment was the DHAP regimen, consisting of high doses of cytarabine and cisplatin. Unfortunately, five months later nodal progression on second-line treatment was detected and the patient was readmitted for further treatment.

On admission to the clinic, computed tomography scan of the chest showed disease progression in mediastinum, both axillae and exudate in the left pleural cavity. Because of danger of central nervous system (CNS) involvement, the patient received 50 mg of liposomal formulation of cytarabine intrathecally as prophylaxis. On the next day, R-IGEV chemotherapy was started, consisting of rituximab $375 \mathrm{mg} / \mathrm{m}^{2}$ on day 1 , Ifosfamide $2000 \mathrm{mg} / \mathrm{m}^{2}$ on days 1 to 4 , gemcitabine $800 \mathrm{mg} / \mathrm{m}^{2}$ on days 1 and 4 , vinorelbine $20 \mathrm{mg} / \mathrm{m}^{2}$ on day 1 , and prednisolone $100 \mathrm{mg}$ on days 1 to 4 [1]. On the second day of Ifosfamide administration the patient developed progressive neurological symptoms including confusion, transient agitation, and involuntary movements of upper extremities. On neurological examination, no focal neurological deficits were detected and computed tomography scan of the head also did not reveal any pathological lesions. Ifosfamide-induced encephalopathy (IIE) was suspected. The infusion of the drug was ceased; hydratation and forced diuresis were implemented. Another drugs with CNS-depressing activity (mainly opioid analgesics) were discontinued or reduced. On the next day, the neurological dysfunction worsened. The quantitative consciousness abnormalities escalated and catatonic symptoms, as well as fecal and urinary incontinence occurred. The vital signs were normal. Laboratory tests revealed moderately increased liver aminotransferase and gammaglutamyltransferase activity, as well as increased serum creatinine and C-reactive protein concentration. Urine analysis disclosed moderate leukocyturia, microhematuria and proteinuria. However, no significant abnormalities of electrolytes, ammonia and glucose serum concentrations were noted. Due to progression of symptoms in spite of the implemented measures, the decision was made to administer methylene blue (MB). The drug was administered intravenously in doses of 50 mg dissolved in $100 \mathrm{ml}$ of normal saline, in 10-minute infusions repeated every 6 hours. Already after several hours from the first dose of MB, resolution of catatonic symptoms and improvement of consciousness status were observed. After the second MB dose neurologic symptoms disappeared almost completely. Consequently, the treatment was stopped. The magnetic resonance imaging (MRI) of the CNS performed several days later showed no focal abnormalities, no diffusion abnormalities nor pathologic contrast enhancements. The liver and kidney function abnormalities resolved during the hospitalization. The patient was discharged from hospital without neurological symptoms. 
www.tridhascholars.org | July-2018

\section{Discussion}

Many cytotoxic agents used in the treatment of hematopoietic neoplasms and solid tumors are characterized by direct or indirect neurotoxic activity. Ifosfamide is an example of these agents - an oxazaphosphorine class alkylating cytotoxic agent. Ifosfamide is a pro-drug requiring enzymatic activation to biologically active metabolites, part of which cross the brain-blood barrier and exhibit toxicity in the central nervous system (CNS).

Ifosfamide-induced encephalopathy (IIE) is a complication noted in $10-40 \%$ of treated patients. It is usually mild or moderate in intensity (grade 1 or 2 according to National Cancer Institute Common Toxicity Criteria-NCI CTC) and has transient course, though severe forms leading to death have also been described (NCI CTC grade 3 or 4) [2,3].

Even though the precise pathogenesis of IIE is unknown, most probably it consists in inhibition of mitochondrial oxidative phosphorylation by Ifosfamide metabolites (mainly chloroacetaldehyde-CAA, chloroethylamine), their direct neurotoxic activity as well as in decreasing the availability of glutathione in the CNS and thus exposing the CNS to intense oxidative stress [4].

Some factors are believed to increase the risk of IIE occurrence and include above all hypoalbuminemia, hypobilirubinemia, anemia, renal insufficiency, electrolyte imbalance, female gender, as well as treatment with agents possessing sedative effect on CNS [5]. Some of the above factors were present in the described case (hypoalbuminemia, anemia, abnormal renal function parameters and concomitant use of opioid analgesics), thus the patient had an increased risk of neurologic complications. However, no association between Ifosfamide dose and IIE incidence has been observed [4].

The clinical picture of the syndrome is diverse and dependent upon the severity of encephalopathy. First symptoms may occur after several hours to days from Ifosfamide initiation. Quantitative disorders of consciousness prevail, ranging from somnolence to coma. Other less frequently observed symptoms are: impairment of memory and spatial orientation, acute psychotic depression, confusion, hallucinations, as well as fecal and urinary incontinence. Motor disorders may also occur, mainly generalized myoclonus and asterixis [6-8].

The diagnosis of IIE is made on the basis of the clinical picture, after exclusion of other possible causes of observed symptoms [8]. The differential diagnosis should include above all encephalopathy caused by metabolic deregulation, the influence of other toxic agents (including drugs), neuroinfection, as well as organic CNS disease. Therefore, the physical examination should be supplemented by a range of additional investigations (including blood biochemistry-liver and kidney function parameters, blood glucose level, electrolytes concentrations, inflammation markers and arterial blood gases), neuroimaging studies (computed tomography, magnetic resonance imaging), and occasionally also toxicologic investigations. Some patients present with abnormal electroencephalographic results, though they are not specific for IIE.

In the presented case the above algorithm was implemented to exclude other causes of encephalopathy. The overall clinical

picture pointed to the diagnosis of IIE, NCI-CTC grade 3, with dominant catatonia and fecal/urinary incontinence. Was the diagnosis confirmed, chemotherapy was consequently withdrawn and interventions to restore proper CNS function were undertaken. 
www.tridhascholars.org | July-2018

The recognized management of IIE includes prompt discontinuation of Ifosfamide infusion, intravenous hyper-hydration and forced diuresis, as well as careful cessation of all other drugs potentially suppressing the CNS function. In most cases such measures are sufficient to reduce the neurologic symptoms in 2-3 days. Nevertheless, the diagnosis of IIE should be made as fast as possible.

As mentioned before, in a subgroup of patients encephalopathy may have severe, sometimes even life threatening clinical course (as in the described case). On these occasions additional therapeutic measures should be considered.

There are reports of high efficacy, early onset of action and relative safety of methylene blue (MB) in IIE treatment. However, the majority of data come from case reports and retrospective case series. Importantly, there are no placebo-controlled clinical trials assessing MB in treatment or prevention of Ifosfamide-induced neurologic symptoms [9-11].

The mechanism of action of this lipophilic dye as a specific antidote to Ifosfamide is not precisely known. It is assumed that MB has multiple cellular and molecular targets, which may antagonize the neurotoxic action of Ifosfamide metabolites, as well as inhibit their generation. It has been confirmed that MB functions as an accessory electron acceptor in mitochondrial electron transport chain and may substitute for inactivated flavoproteins, thus improving the deteriorated mitochondrial oxidative phosphorylation process. Moreover, MB neutralizes free radicals, inhibits serum and extrahepatic monoamine oxidases, as well as modulates the cyclic guanosine monophosphate (cGMP) signalling pathway [12-15].

No severe adverse reactions or complications of MB administration have been reported so far. On the contrary, spectacularly fast regression of encephalopathy was observed (during up to 10 minutes). The most frequently applied dosage was 50 mg every 6 hours continued until regression of neurologic symptoms. Both the intravenous route (dye solution was diluted in 100 $\mathrm{ml}$ of $0.9 \%$ saline and administered in 10 minutes) and the oral route of administration were exploited. Nevertheless it is believed that intravenous MB infusion enables much higher CNS concentration of the dye [16,17].

Taking into consideration the severe course of IIE in the described case, unresolving after the cessation of Ifosfamide, a decision was made to infuse MB intravenously in the dosage most frequently used in published reports $(50 \mathrm{mg} \mathrm{in} 100 \mathrm{ml}$ of $0.9 \% \mathrm{NaCl})$. This management proved to be clinically efficacious with considerable improvement (alleviation of catatonic symptoms) already after the first dose, and almost complete resolution of neurologic symptoms about 4 hours after the second dose. There was no need to give the third dose and the patient left the ward without any clinical neurologic symptoms of IIE. Even though, Ifosfamide-based chemotherapy was never introduced in this patient again, because a history of severe IIE is a contraindication to continued treatment with this cytotoxic agent.

If IIE symptoms would persist despite the above mentioned interventions, administration of thiamine in high doses (100 mg intravenously every 6 hours) could be considered. There are data from case reports suggesting the efficacy of such treatment with neurologic improvement after 30 hours [18-20].

In the current case, intrathecal infusion might have contributed to the development and severity of IIE Administration of liposomal cytarabine one day earlier. While there is no proof that cytarabine potentiated the CNS effects of Ifosfamide it cannot be excluded. The possible effects of such association were not reported earlier and definitely, this association should be avoided in the future. 
www.tridhascholars.org | July-2018

In conclusion, in case of acute neurologic symptoms occurring during Ifosfamide-based chemotherapy, IIE should always be considered. Combination of intrathecal cytarabine and systemic Ifosfamide should be avoided. Once the clinical diagnosis of this complication is made, discontinuation of Ifosfamide administration and initiation of symptomatic treatment is mandatory. In severe forms of IIE administration of MB is highly advisable, that proved to be safe and efficacious in the described patient. Due to insufficient data, currently MB should not be administered routinely in IIE prophylaxis.

\section{References}

1. Santoro A, Magagnoli M, Spina M, et al. (2007) Ifosfamide, gemcitabine, and vinorelbine: a new induction regimen for refractory and relapsed Hodgkin's lymphoma. Haemetologica 92: 35-41.

2. Sweiss KI, Beri R, Shord SS (2008) Encephalopathy after high-dose Ifosfamide: a retrospective cohort study and review of the literature. Drug Safety 31(11): 989-996.

3. Shin YJ, Kim JY, Moon JW, et al. (2011) Fatal Ifosfamide-induced metabolic encephalopathy in patients with recurrent epithelial ovarian cancer: report of two cases. Cancer Research and Treatment: Official Journal of Korean Cancer Association 43(4): 260-263.

4. Ajithkumar T, Parkinson C, Shamshad F et al. (2007) Ifosfamide encephalopathy. Clinical Oncology 19(2): 108-114.

5. Rieger C, Fiegl M, Tischer J, et al. (2004) Incidence and severity of Ifosfamide-induced encephalopathy. Anti-Cancer Drugs 15(4): 347-350.

6. http://baxter.com.pl/downloads/charakterystyki/Oncology/Endoxan_1\%20g.pdf.

7. Savica R, Rabinstein AA and Josephs KA (2011) Ifosfamide associated myoclonus-encephalopathy syndrome. Journal of Neurology 258(9): 1729-1731.

8. Klastersky J (2003) Side effects of Ifosfamide. Oncology 65(Suppl. 2): 7-10.

9. Patel PN (2006) Methylene blue for management of Ifosfamide-induced encephalopathy. Annals of Pharmacotherapy 40(2): 299-303.

10. Pelgrims J, De Vos F, Van den Brande J, et al. (2000) Methylene blue in the treatment and prevention of Ifosfamideinduced encephalopathy: report of 12 cases and a review of the literature. British Journal of Cancer 82(2): 291-294.

11. Brunello A, Basso U, Rossi E, et al. (2007) Ifosfamide-related encephalopathy in elderly patients. Drugs \& Aging 24(11): 967-973.

12. Oz M, Lorke DE, Hasan M, et al. (2011) Cellular and molecular actions of methylene blue in the nervous system. Medicinal Research Reviews 31(1): 93-117.

13. Aeschlimann C, Cerny $\mathrm{T}$ and Küpfer A (1996) Inhibition of (mono) amine oxidase activity and prevention of Ifosfamide encephalopathy by methylene blue. Drug Metabolism and Disposition 24(12): 1336-1339.

14. Küpfer A, Aeschlimann C and Cerny T (1996) Methylene blue and the neurotoxic mechanisms of Ifosfamide encephalopathy. European Journal of Clinical Pharmacology 50(4): 249-252.

15. Furian AF, Fighera MR, Oliveira MS, et al. (2007) Methylene blue prevents methylmalonate-induced seizures and oxidative damage in rat striatum. Neurochemistry International 50(1): 164-171.

16. Ferrero JM, Eftekari P, Largillier R, et al. (1995) Treatment of Ifosfamide induced encephalopathy with methyleneblue. Bulletin du Cancer 82(7): 598-599.

17. Peter C, Hongwan D, Küpfer A, et al. (2000) Pharmacokinetics and organ distribution of intravenous and oral methylene blue. European Journal of Clinical Pharmacology 56(3): 247-250. 
www.tridhascholars.org | July-2018

18. Imtiaz S and Muzaffar N (2010) Ifosfamide neurotoxicty in a young female with a remarkable response to thiamine. JPMA. The Journal of the Pakistan Medical Association 60(10): 867-869.

19. Hamadani M and Awan F (2006) Role of thiamine in managing Ifosfamide-induced encephalopathy. Journal of Oncology Pharmacy Practice 12(4): 237-239.

20. Buesa JM, García-Teijido P, Losa R, et al. (2003) Treatment of Ifosfamide encephalopathy with intravenous thiamin. Clinical Cancer Research 9(12): 4636-4637. 